\title{
Quantitative characterization of nanometer-scale electric fields via momentum- resolved STEM
}

Andreas Beyer ${ }^{1}$, Saleh Firoozabadi ${ }^{2}$, Damien Heimes ${ }^{1}$, Tim Grieb ${ }^{3}$, Andreas Rosenauer ${ }^{4}$, Knut MüllerCaspary $^{5}$ and Kerstin Volz ${ }^{1}$

${ }^{1}$ Materials Science Centre and Department of Physics, Philipps University Marburg, Hans-MeerweinStraße 6, Marburg, 35032, Germany, Germany, ${ }^{2}$ Materials Science Centre and Department of Physics, Philipps University Marburg, Hans-Meerwein-Straße 6, Marburg, 35032, Germany, Marburg, Hessen, Germany, ${ }^{3}$ 2Institut für Festkörperphysik, Universität Bremen, Otto-Hahn-Allee 1, Bremen, 28359, Germany, United States, ${ }^{4}$ Institut für Festkörperphysik, Universität Bremen, Otto-Hahn-Allee 1, Bremen, 28359, Germany, United States, ${ }^{5}$ Ernst Ruska Centre for Microscopy and Spectroscopy with Electrons and the Peter Grünberg Institute Forschungszentrum Jülich, 52425 Jülich, Germany, United States

The functionality of modern nano-scale devices such as transistors, solar cells and batteries relies on builtin electric fields. These fields can have an extension of several tens of nanometers and strengths of several $\mathrm{MV} / \mathrm{cm}$. At the same time, unwanted, parasitic fields in the same order of magnitude can lead to a device's failure. Accordingly, the detection and quantification of such fields are highly desired.

Scanning transmission electron microscopy (STEM) provides a suitable high spatial resolution for this task. Recently, four-dimensional STEM (4DSTEM) has been shown to be capable of measuring electric fields within a sample. More precisely, momentum-resolved STEM (MRSTEM) facilitates measuring the shift of the center-of-mass (COM) of the diffracted intensity. This shift is directly proportional to the strength of the electric field present (Müller-Caspary et al., 2017). So far, MRSTEM has been applied mainly to address the fields introduced by the atomic columns of the sample atoms, which requires TEM samples as thin as $5 \mathrm{~nm}$ (Ophus, 2019). Here, we address longer-range fields present in actual devices. Since they are several orders of magnitude smaller than atomic electric fields, much higher TEM-sample thicknesses are required to introduce a notable shift to the COM.

To investigate the capabilities of the field detection method, we use two GaAs-based pn junctions with different defined electric fields determined by the dopant concentrations on both sides of a junction. We utilize advanced focused ion beam (FIB) preparation in a JEOL JIB 4601 resulting in defined thickness steps between 150 and $350 \mathrm{~nm}$, followed by a low energy Argon ion milling in a Fischione Nanomill to reduce the presence of inactive, amorphous layers on the sample's surface. 4DSTEM measurements were carried out in double aberration-corrected JEOL JEM 2200FS operating at $200 \mathrm{kV}$. A pixelated pnCCD detector was used to capture a full diffraction pattern at each scanning point. Complementary simulation of the 4D data was carried out using the STEMsalabim code(Oelerich et al., 2017). Home-written Matlab scripts were used for post-processing of the acquired data and retrieving the electric fields.

We quantitatively derived the key properties of the two pn junctions, such as the polarity of the junction (i.e., pn or $\mathrm{np}$ ), the width of the depletion width, the doping levels on each side of the junctions, as well as the field strengths and the built-in voltages (Beyer et al., 2021). The doping levels derived are in very good agreement with supplementary, non-TEM-based methods, i.e. Hall and electrochemical capacitancevoltage (ECV) measurements. 
The impact of the imaging conditions on the derived quantities, especially at this rather high sample thicknesses is investigated by systematic multislice simulations considering additional electric fields.

The present contribution shows how MRSTEM can be used to quantify electric fields present in actual devices.

References

Beyer, A., Munde, M. S., Firoozabadi, S., Heimes, D., Grieb, T., Rosenauer, A., Müller-Caspary, K. \& VOLZ, K. (2021). Quantitative Characterization of Nanometer-Scale Electric Fields via MomentumResolved STEM. Nano Letters accepted, anolett.0c04544. https://pubs.acs.org/doi/10.1021/acs.nanolett.0c04544.

Müller-Caspary, K., Krause, F. F., Grieb, T., Löffler, S., Schowalter, M., Béché, A., Galioit, V., Marquardt, D., Zweck, J., Schattschneider, P., Verbeeck, J. \& Rosenauer, A. (2017). Measurement of atomic electric fields and charge densities from average momentum transfers using scanning transmission electron microscopy. Ultramicroscopy 178, 62-80. http://linkinghub.elsevier.com/retrieve/pii/S0304399116300596.

Oelerich, J. O., DuscheK, L., Belz, J., Beyer, A., Baranovskit, S. D. \& Volz, K. (2017). STEMsalabim: A high-performance computing cluster friendly code for scanning transmission electron microscopy image simulations of thin specimens. Ultramicroscopy 177.

OpHUS, C. (2019). Four-Dimensional Scanning Transmission Electron Microscopy (4D-STEM): From Scanning Nanodiffraction to Ptychography and Beyond. Microscopy and Microanalysis 1-20. https://www.cambridge.org/core/product/identifier/S1431927619000497/type/journal_article. 